\title{
New Routine for Nuclear Medicine Technologists to Determine When to Add SPECT/CT to a Whole-Body Bone Scan
}

Asal Shafi, Ola Thorsson, and Lars Edenbrandt

Clinical Physiology and Nuclear Medicine, Skåne University Hospital, Lund University, Malmö, Sweden

\begin{abstract}
Bone scintigraphy is usually obtained as a whole-body scan producing 2 images: an anterior view and a posterior view. Sometimes abnormal findings in the spine are difficult to distinguish on whole-body bone scans. SPECT/CT may be performed to localize and interpret a lesion correctly and to help differentiate between benign and metastatic lesions. The assessment of whether SPECT/CT is needed is usually made by a physician. The aim of this study was to evaluate our new routine for nuclear medicine technologists to determine when to add SPECT/CT to whole-body bone scintigraphy. Methods: A 3-part educational course was developed for the nuclear medicine technologists. The first part was to learn criteria for when SPECT/CT should be added to a whole-body bone scan. The second part was to review a selection of training whole-body bone scans illustrating the criteria. The third part was to pass a test of whether whole-body bone scans should be supplemented by SPECT/CT. Results: The nuclear medicine technologists and the physicians agreed that SPECT/CT was required in 63 cases and not required in 27 cases. The resulting percentage agreement was $90 \%$, and the $\kappa$ value was 0.77 . There was disagreement in 10 cases. In 6 of these cases only the nuclear medicine technologists wanted to add SPECT/CT, and in 4 of these cases only the physicians wanted to add SPECT/CT. Conclusion: After participating in the training course developed in this project, the nuclear medicine technologists were able to decide whether a SPECT/CT study is needed. An implication of this result is that the effectiveness of the nuclear medicine department should be improved after our new routine is implemented. The successful outcome of this project may stimulate departments to take on similar quality-improvement projects in the future.
\end{abstract}

Key Words: assessment; criteria; whole-body bone scan; SPECT/CT; image interpretation

J Nucl Med Technol 2014; 42:28-32

DOI: 10.2967/jnmt.113.132035

B one scintigraphy, one of the most frequently performed nuclear medicine procedures in most nuclear medicine departments, has been shown to be sensitive in identifying

Received Oct. 30, 2013; revision accepted Dec. 10, 2013.

For correspondence or reprints contact: Asal Shafi, Clinical Physiology and Nuclear Medicine, Skåne University Hospital-Malmö, SE-205 02 Malmö, Sweden. E-mail: asal_shafi@hotmail.com

Published online Jan. 27, 2014.

COPYRIGHT (c) 2014 by the Society of Nuclear Medicine and Molecular Imaging, Inc. skeletal metastatic lesions $(1-3)$. The main indication for bone scintigraphy is to detect bone metastases as early as possible and to evaluate problems with malignant bone involvement such as pathologic fractures or degenerative changes $(4,5)$. The most common localization site of malignant tumors is the skeleton, followed by the lungs and the liver (6). Love et al. demonstrated that about $75 \%$ of patients with skeletal pain show abnormal uptake on bone scintigraphy and that $25 \%$ $45 \%$ of asymptomatic patients with malignancy have bone metastasis findings (7).

Bone scintigraphy is usually obtained as a whole-body scan producing 2 images: an anterior view and a posterior view. Abnormal lesions in the bone scans appear as areas of either increased or decreased intensity. These findings are, however, nonspecific and may have causes other than tumors, such as fractures and degenerative disease. Sometimes, abnormal findings in the spine are difficult to distinguish on whole-body bone scans. SPECT/CT may be performed to localize and interpret a lesion correctly and to help differentiate between benign and malignant lesions $(8,9)$. Horger et al. concluded that SPECT/CT improves the diagnostic precision of whole-body bone scans by identifying benign bone abnormalities (10). In addition, some abnormal findings can be detected only by using tomographic techniques. The spine is the most common site of skeletal metastases (11). Therefore, optimal interpretation of skeletal lesions in this region is important. SPECT/CT provides more anatomic information than whole-body bone scans and detects $20 \%-50 \%$ more lesions in the spine $(12,13)$. Iqbal et al. showed that the addition of SPECT/CT significantly reduced the number of false-negative results and increased the number of true-positive results in a study of 80 patients with a solitary lesion in the spine on wholebody bone scans (14).

Modern SPECT/CT systems combine a double-head gamma camera with a CT scanner within the same gantry. With this type of hybrid camera, a whole-body bone scan and a CT examination can be performed immediately after each other with the patient in the same position. Whether a SPECT/CT scan is needed is usually assessed by physicians, who commonly are busy in other parts of the hospital at the time when such a decision should be made. A better patient experience and quicker throughput would result if the decision were made immediately by the nuclear medicine technologist. The technologist who acquires 
and reviews the images for quality assurance then would not have to wait for a decision by the physician, and the physician would have more time to interpret the bone scintigraphy and SPECT/CT images.

In order to allow technologists to decide whether SPECT/CT is required, we developed a new routine in which each technologist first learns our criteria for adding SPECT/CT to bone scanning, then reviews 40 training cases, and finally passes a test of 10 cases. The aim of this study was to evaluate the new routine.

\section{MATERIALS AND METHODS}

\section{Study Design}

A 3-part educational course was developed for nuclear medicine technologists (technologists with specific training in nuclear medicine and CT as part of their formative professional education). The first part was to learn criteria for adding SPECT/ CT to a whole-body bone scan. The second part was to review a selection of training whole-body bone scans illustrating the criteria. The third part was to pass a test of when whole-body bone scans should be supplemented by SPECT/CT. Nuclear medicine technologists who passed the course then became authorized to determine when to add SPECT/CT to whole-body bone scintigraphy. The new routine was evaluated by letting nuclear medicine technologists and physicians responsible for clinical routine cases independently decide whether SPECT/CT was required. The evaluation group consisted of 100 consecutive patients.

\section{Part 1: Criteria}

We developed criteria for when SPECT/CT should be added to a whole-body bone scan. The criteria are based on findings on the current bone scan, a comparison with the previous scan, and pain or injury reported by the patient. The criteria are applied only to patients with known or suspected malignant disease who are undergoing routine bone scanning for screening or follow-up. For other indications, the nuclear medicine physician makes the decision regarding SPECT/CT (Table 1).

\section{Part 2: Training Cases}

We selected 40 training cases to illustrate the criteria. The cases consisted of whole-body bone scans from patients with malignant disease together with clinical information from the referral and symptoms reported by the patients. Custom bone software based on EXINI (EXINI Diagnostics AB) was developed to present the images, the clinical information, and the desired decision regarding SPECT/CT (Fig. 1).

\section{Part 3: Test Cases}

Ten test cases were selected in the same way as the training cases. The cases were presented in the same way as the training cases except that the desired decision was not available. The nuclear medicine technologists reviewed the test cases and made the decision on the basis of the criteria and their experience from the training cases (Fig. 2).

\section{Evaluation in Clinical Routine}

The new routine was evaluated by comparing the decisions made by the nuclear medicine technologists and the decisions made by the physicians for a group of 100 consecutive patients in clinical routine. The nuclear medicine technologist responsible for each whole-body bone scan decided whether a SPECT/CT study was required before asking the physician. The physician's decision was made without knowledge of the technologist's decision. Six nuclear medicine technologists and 4 physicians were involved in the study. Both the physicians and the nuclear medicine technologists had access to clinical and referral information.

\section{Patients}

The 40 training cases and 10 test cases were selected from patients who underwent a routine whole-body bone scan between 2009 and 2011. The evaluation in clinical routine was based on 100 patients who underwent a routine whole-body bone scan between August and October 2011 at Skåne University Hospital, Malmö, Sweden. Patients with known malignant disease who were undergoing routine bone scanning for screening or follow-up were included in the study. The mean age was $69 \pm 11 \mathrm{y}$. According to Swedish law, a study regarded as quality-improvement work does not need formal approval from a local research ethics committee.

TABLE 1

Criteria for Performing SPECT/CT After Whole-Body Bone Scan

\begin{tabular}{ll}
\hline Section & Description \\
\hline A Indications for not performing SPECT/CT after bone scan \\
Patient cannot participate in SPECT/CT examination \\
Widespread metastatic disease is present \\
A previous whole-body bone scan is available and no new lesions are present \\
B Indications for performing SPECT/CT after bone scan \\
Focal lesions in spine or pelvis are present \\
Patient reports newly developed pain from spine or pelvis \\
Prostate cancer patients have prostate-specific antigen that is increasing or $>20 \mathrm{ng} / \mathrm{mL}$ even though no metastatic lesions \\
are present (SPECT/CT of lumbar spine and pelvis) \\
Large urinary bladder is covering sacrum and patient has reported symptoms from this area \\
C Indications for not performing SPECT/CT \\
Whole-body bone scan has normal results and no criteria from section B to perform SPECT/CT are fulfilled
\end{tabular}

These criteria apply to patients suspected of having metastatic disease. For other indications, nuclear medicine physician makes decision. 
FIGURE 1. Training cases. Bone scan images are displayed on left. Clinical information from referral and symptoms from patient are on right. Desired decision "SPECT/CT required or not" is displayed only after technologist has made decision and has clicked button to show this information.

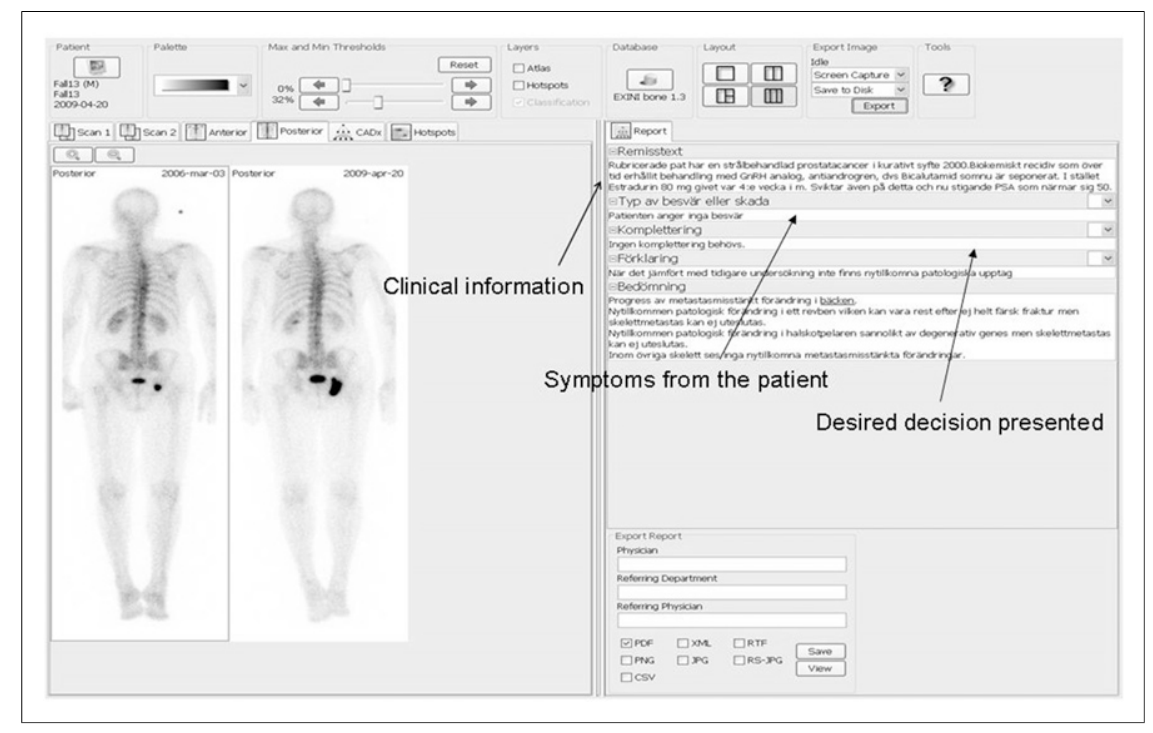

The study was performed in accordance with the principles of the Declaration of Helsinki.

\section{Bone Scintigraphy}

Whole-body bone scans were obtained $3 \mathrm{~h}$ after intravenous injection of $600 \mathrm{MBq}$ of ${ }^{99 \mathrm{~m}} \mathrm{Tc}$-hydroxymethylene diphosphonate. The images were obtained according to established clinical protocols, using a Symbia T2 (Siemens AG Medical Solutions) SPECT/CT hybrid gamma camera. This system includes a dualdetector gamma camera combined with a 2-slice CT scanner for attenuation correction and anatomic information. The images were visualized in both E.soft (Siemens AG Medical Solutions) and EXINI bone software package. SPECT/CT images were obtained according to established clinical protocols, using the same Symbia T2 gamma-camera system. SPECT imaging was performed using a low-energy high-resolution collimator. The CT images were obtained within the same gantry on a $128 \times 128$ matrix, with a voltage of $130 \mathrm{kV}$, tube current of $20 \mathrm{~mA}$, and with a $5.0-\mathrm{mm}$ slice thickness.

\section{Statistical Methods}

To evaluate agreement between nuclear medicine technologists and physicians in their decisions about the addition of SPECT/CT, the $\kappa$ statistic was used. Statistics were performed using SPSS (version 16.0; SPSS Inc.).

\section{RESULTS}

The nuclear medicine technologists and physicians agreed that SPECT/CT was required in 63 cases and not required in 27 cases, as presented in Table 2 . The resulting percentage agreement was $90 \%$, and the $\kappa$ value 0.77 . There was disagreement in 10 cases. In 6 cases, only the nuclear medicine technologists wanted to add SPECT/CT, and in 4 cases, only the physicians wanted to add SPECT/ CT. The 10 cases of disagreement were reevaluated in a masked manner by the most experienced physician at

FIGURE 2. Test cases. Images are displayed in same way as for training cases except that desired decision regarding "SPECT/CT required or not" is not available.

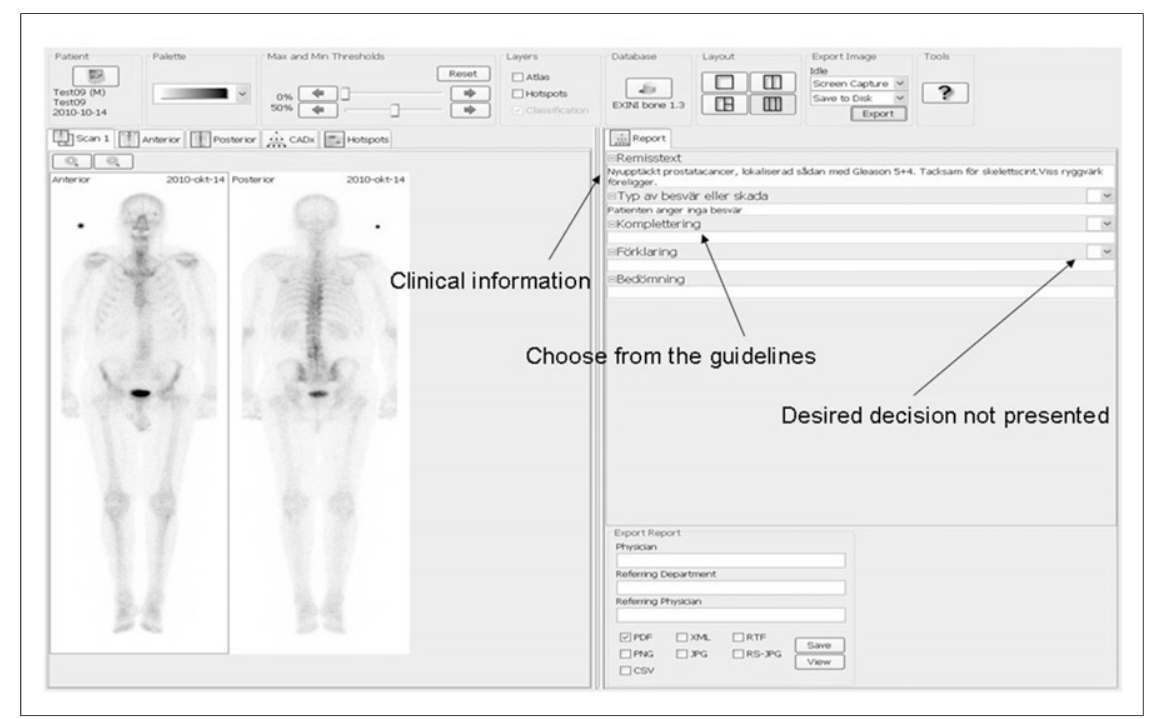


TABLE 2

Distribution of 100 Evaluation Cases from Nuclear Medicine Technologists and Physicians

\begin{tabular}{lcc}
\hline & \multicolumn{2}{c}{ Nuclear medicine technologist } \\
\cline { 2 - 3 } Physician & $\begin{array}{c}\text { SPECT/CT } \\
\text { required }\end{array}$ & $\begin{array}{c}\text { SPECT/CT not } \\
\text { required }\end{array}$ \\
\hline $\begin{array}{l}\text { SPECT/CT } \\
\text { required }\end{array}$ & 63 & 4 \\
$\begin{array}{l}\text { SPECT/CT not } \\
\text { required }\end{array}$ & 6 & 27 \\
\hline
\end{tabular}

our department, and his decision in all cases was that SPECT/CT was not required.

\section{DISCUSSION}

The results of this study show that nuclear medicine technologists, after a period of formal training, are able to decide when an additional SPECT/CT examination is necessary after a whole-body bone scan. The agreement between the decisions made by the nuclear medicine technologists and the decisions made by the physicians was good in the group of 100 evaluation cases. No previous study has, to our knowledge, evaluated this routine. Johansson et al. assessed whether nuclear medicine technologists were able to decide whether a rest study was needed after stress myocardial perfusion scintigraphy (15). In their study, 3 nuclear medicine technologists and 3 physicians individually categorized each of 532 stress studies as "rest study required" or "no rest study required." They concluded that it is possible to delegate this assessment to the nuclear medicine technologists and that such delegation could improve the effectiveness of a nuclear medicine department. As in our study, the risk that this assessment would be incorrect was not higher for the nuclear medicine technologists than it was for the physicians.

In our study, several precautions were made to minimize the risk of sending a patient home without performing a necessary SPECT/CT examination. First, the technologists' decision was based on criteria established jointly by the physicians and the technologists in the department. Also, the new routine was applied only to scans with metastasis as the indication; for other referral indications, the technologist always consulted a physician. All cancers have different metastatic behaviors, and it would have been possible to limit the scope of the new routine to, for example, prostate and breast cancer, but we decided to include all scans with metastasis as the indication. Finally, if the nuclear medicine technologist feels uncertain whether SPECT/CT is required even with the help of given criteria, the individual still has the opportunity to consult the physician for a decision. In the study of Johansson et al. (15), all technologists and physicians had to classify all cases and there was no possibility to abstain from a decision. Our approach is closer to the clinical routine, in which there generally is a possibility for the technologist to discuss with a physician if in doubt.

On January 24, 2012, the routine used in our study was implemented for the nuclear medicine technologists at the department of Clinical Physiology, Skåne University Hospital, Malmö. It is our impression that the effectiveness of our nuclear medicine department has indeed improved. As expected, the nuclear medicine technologist generally does not have to wait for a decision by the physician, and the physician has more time to interpret the bone scintigraphy and SPECT/CT images. The work to develop the criteria, the training cases, and the test cases, and their use in clinical routine, also benefits the department by helping to ensure that correct decisions are made independently of which individual technologist or physician is deciding. An interesting side effect is that the training and test cases used in the training course for this routine can also be used in the general education of new nuclear medicine technologists and physicians in the department. We have found that using this approach increases the efficiency of the training process and lessens the dependence on individual teaching skills. In addition, there is interobserver variability between different physicians. Sadik et al. reported only moderate interobserver agreement ( $\kappa$ ranged from 0.16 to 0.82 ) when 37 observers from 18 hospitals were compared pairwise regarding interpretation of bone scans (16). In a review, Owen et al. encourage the use of protocols to maximize care and management by minimizing inter- and intraprofessional variation (17).

Health care costs can be reduced if additional SPECT/CT images are acquired only when they have clinical value. In our environment, adding SPECT/CT is associated with greater costs due to increased investigation time and time to interpret the images. The addition of $\mathrm{CT}$ to the nuclear medicine procedure usually incurs only a small additional dose (e.g., $1.0 \mathrm{mSv}$ ), but more significant doses can be delivered, depending on the CT technology that is used. The decision to add SPECT/CT should therefore be based on knowledge and experience in this field as expressed in comprehensive criteria. In general, we feel that the experience from this study can be useful for future projects in other areas of imaging.

Our study had some limitations. The number of training and especially test cases should ideally be larger to improve training and make the test results more accurate. In our department, however, it has been difficult to find more time for this type of task for nuclear medicine technologists and physicians in the clinical routine.

Some SPECT/CT systems now have high-end diagnosticquality CT systems. In this study, we used low-dose CT. The radiation dose to the patient is higher for conventional CTand this factor has to be considered — but the general results from this study would be applicable also in a clinical setting with conventional CT. 


\section{CONCLUSION}

There was good agreement in this study between nuclear medicine technologists and physicians in assessing whether SPECT/CT was required in addition to whole-body bone scans. After participating in the training process developed in this project, the nuclear medicine technologists were able to decide whether a SPECT/CT study is needed. An implication of this result is that the effectiveness of the nuclear medicine department should be improved after our new routine is implemented. The successful outcome of this project may stimulate departments to take on similar qualityimprovement projects in the future.

\section{DISCLOSURE}

Lars Edenbrandt is employed by and is a stockholder of EXINI Diagnostics AB, Lund, Sweden. No other potential conflict of interest relevant to this article was reported.

\section{REFERENCES}

1. Jacobson AF, Fogelman I. Bone scanning in clinical oncology: does it have a future? Eur J Nucl Med. 1998;25:1219-1223.

2. Ryan PJ, Fogelman I. Bone scintigraphy in metabolic bone disease. Semin Nucl Med. 1997;27:291-305.

3. Rubenstein J. Imaging of skeletal metastases. Tech Orthop. 2004;19:2-8.

4. Even-Sapir E. Imaging of malignant bone involvement by morphologic, scintigraphic and hybrid modalities. J Nucl Med. 2005;46:1356-1367.
5. Currie GM, Haase M, Hashmi R, Kiat H. Hormone therapy in prostate cancer. J Nucl Med Technol. 2013;41:49-51.

6. Savelli G, Maffiolli L, Maccauro M, De Deckere E, Bombardieri E. Bone scintigraphy and the added advantage of SPECT in detecting skeletal lesions. $Q J$ Nucl Med. 2001;45:27-37.

7. Love C, Din A, Tomas M, Kalapparambath T, Palestro C. Radionuclide bone imaging: an illustrative review. Radiographics. 2003;23:341-358.

8. Utsunomiya D, Shiraishi S, Imuta M, et al. Added value of SPECT/CT fusion in assessing suspected bone metastasis: comparison with scintigraphy alone and non-fused scintigraphy and CT. Radiology. 2006;238:264-271.

9. Horger M, Bares R. The role of single-photon emission computed tomography/ computed tomography in benign and malignant bone disease. Semin Nucl Med. 2006;36:286-294.

10. Horger M, Eschmann SM, Plannenberg C, et al. Evaluation of combined emission and transmission tomography for classification of skeletal lesions. AJR. 2004;183:655-661.

11. Taoka T, Mayr N, Lee H, et al. Factors influencing visualization of vertebral metastases on MR imaging versus bone scintigraphy. AJR. 2001;176:1525-1530.

12. Gates GF. SPECT bone scanning of the spine. Semin Nucl Med. 1998;28:78-94.

13. Papathanassiou D, Bruna-Muraille C, Jouannaud C, Gagneux-Lemoussu L, Eschard J, Liehn J. Single-photon emission computed tomography combined with computed tomography (SPECT/CT) in bone diseases. Joint Bone Spine. 2009;76:474-480.

14. Iqbal B, Currie GM, Wheat JM, Raza H, Kiat H. The incremental value of SPECT/CT in characterizing solitary spine lesions. J Nucl Med Technol. 2011;39:201-207.

15. Johansson L, Lomsky M, Gjertsson P. Can nuclear medicine technologists assess whether a myocardial perfusion rest study is required? J Nucl Med Technol. 2008;36:181-185.

16. Sadik M, Suurkula M, Höglund P, Järund A, Edenbrandt L. Quality of planar whole-body bone scan interpretations: a nationwide survey. Eur J Nucl Med Mol Imaging. 2008;35:1464-1472.

17. Owen A, Hogg P, Nightingale J. A critical analysis of a locally agreed protocol for clinical practice. Radiography. 2004;2:139-144. 